\begin{tabular}{ll}
\hline Frontiers in Heat and Mass Transfer \\
\hline
\end{tabular}

\title{
A HYBRID CELLULAR AUTOMATON METHOD FOR STRUCTURAL TOPOLOGY OPTIMIZATION WITH MECHANICAL AND HEAT CONSTRAINTS
}

\author{
Xiaolei Deng ${ }^{\mathrm{a}, \mathrm{b}, \mathrm{c}, * \dagger}$, Jin Wang ${ }^{\mathrm{d}}$, Jinyu Zhou ${ }^{\mathrm{a}}$, Hongcheng Shen ${ }^{\mathrm{a}}$, Zefeng Sheng ${ }^{\mathrm{a}}$, \\ Jianglin Zhang ${ }^{\mathrm{a}}$, Xiaowen $\mathrm{Lv}^{\mathrm{a}}$ and Changxiong $\mathrm{Xie}^{\mathrm{a}}$
}

\author{
${ }^{a}$ Key Laboratory of Air-driven Equipment Technology of Zhejiang Province, Quzhou University, Quzhou, Zhejiang, 324000, China \\ ${ }^{b}$ Key Laboratory of $3 D$ Printing Process and Equipment of Zhejiang Province, Zhejiang University, Hangzhou, Zhejiang, 310027, China \\ ${ }^{c}$ Zhejiang Yonglida CNC Technology Co., Ltd., Quzhou, Zhejiang, 324000, China \\ ${ }^{d}$ College of Engineering, Southwest Petroleum University, Nanchong, Sichuan, 637800, China
}

\begin{abstract}
A hybrid cellular automaton model combined with finite element method for structural topology optimization with mechanical and heat constraints is developed. The effect of thermal stress on structural optimization is taken into account. Higher order 8-node element and von Neumann strategy are employed for the finite element and the cellular element, respectively. The validating studies of standard testing structure for topological optimization are carried out. The structure evolution, stress evolution and thermal evolution of topology optimization with mechanical and heat constraints are investigated. The results show the developed hybrid method is more efficient for structural topology optimization. Meanwhile, the topology optimization can eliminate most of the thermal stress in the structure.
\end{abstract}

Keywords: Hybrid Cellular Automaton Model, Structure with Mechanical and Heat Constraints, Topology Optimization

\section{INTRODUCTION}

Topological optimization of continuum structure (TOCS) has drawn attentions of many researchers due to its importance and wide application in various fields such as new materials (Czubacki et al. (2015), Bendsoe et al. (2010)) mechanical manufacture (Sigmund et al. (2009)) and biomechanics (Pettermann et al. (1997)). During these engineering processes, this type of optimization for continuum structure presents decisive choice of the appropriate topology of a structure with some constraints in the conceptual phase of a novel product (Eschenauer et al. (2001)). Over the last few decades, various advanced models and methods for TOCS have been explored based on more efficient cellular automaton (CA) algorithm.

Sanaei and Babaei (2011) carried out topology optimization of twodimensional elastic structures with shear and flexural strains by CA model. Zhu and Liu (2000) derived an anisotropic crystalline etching simulation program based on a novel continuous CA algorithm; more efficient and accurate topology optimizations were presented by the developed continuous model. A CA-based algorithm with five-step optimization procedure was proposed for simultaneous shape and topology optimization of continuum structures by Sanaei and Babaei (2012). Du et al. (2013) studied numerical instabilities of topology optimization for continuum structures based on the CA theory.

Despite these recent advances, the nonlinearity and numerical instability of CA algorithm present a significant challenge must be resolved in order to approach the global optimum solution (strain energy) of topology optimization of continuum. Recently, Hybrid Cellular
Automaton Method (HCAM) coupling CA algorithm and Finite Element (FE) model proposed by Tovar et al. $(2004,2005)$ is a no-gradient topology optimization where global strain energy obtained by FE model is used as status information of CA. It's demonstrated that HCAM has the merits of quick convergence and high computing efficiency for topology optimization of continuum (Tovar et al. (2007), Tovar et al. (2006)). Furthermore, Tovar and coworkers investigated crashworthy structures (Bandi et al. (2013)) and piezoelectric material (Lee et al. (2013)) by HCAM combined with controlled energy absorption. Salonitis (2017) studied hybrid CA- FE model for the grind-hardening processing simulation.

In present study, structural topology optimization with mechanical and heat constraints is presented. Structures with mechanical and heat constraints can find its applications in many industries such as metal fabrication and printed circuit board substrates, but studies about thermal topology optimization are seldom involved due to complex mechanisms coupling mechanics and thermotics. Takezawa et al (2014) investigated topology optimization under strength and heat conductivity constraints. It's shown that the effect of thermal expansion on the optimal shapes became more serious under the structure with both sides fixed. Min et al. (2007) suggested a material mixing method based on evolutionary structural optimization for a multiple material structure with multiple thermal constraints. To better understand thermal topology optimization, an efficient computational model based on HCAM for structure with mechanical and heat constraints is developed in the paper. The verifiable investigation of the developed model is carried out for a TOCS standard testing structure.

\footnotetext{
* Quzhou University, Quzhou, Zhejiang, 324000, China

† Corresponding author. Email:dxl@zju.edu.cn
} 


\section{Problem Statement and Formulation}

\subsection{Problem Formulation}

Fig. 1 shows the physical model for a TOCS testing structure with mechanical and heat constraints. The cuboid testing structure with $60 \mathrm{~mm}$ length and $30 \mathrm{~mm}$ width is subject to uniform temperature of $0^{\circ} \mathrm{C}$ on the vertical walls and of $100^{\circ} \mathrm{C}$ on the horizontal walls. As the figure shown, both the vertical walls and four positions with $8 \mathrm{~mm}$ length on the horizontal walls are fixed for the mechanical constraints.

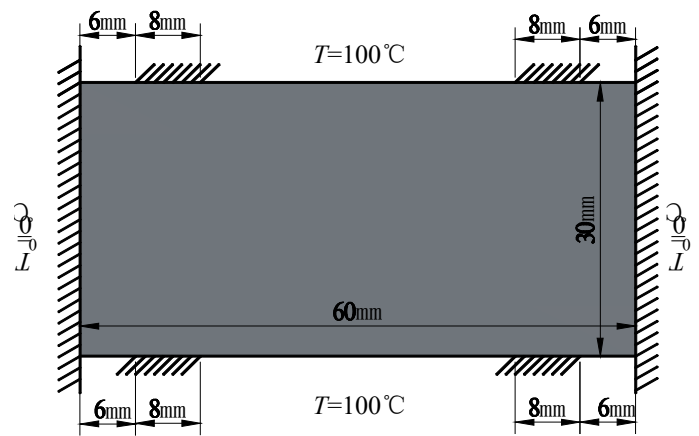

Fig. 1 Physical model for structure with mechanical and heat constraints

Due to thermal stress caused by nonuniform temperature, the performance of the structure with mechanical and heat constraints is changed. Moreover, the thermal stress can exceed the material's yield strength as the temperature increases. Therefore, it should take thermal effects into consideration for structural topology optimization with mechanical and heat constraints. According to the linear thermal stress theory (Kushnir (2009)), the governing equations for the structure with mechanical and heat constraints can be expressed as follows.

$$
\begin{aligned}
& \rho c_{p} \frac{\partial T}{\partial t}=\frac{\partial}{\partial x}\left(\lambda \frac{\partial T}{\partial x}\right)+\frac{\partial}{\partial y}\left(\lambda \frac{\partial T}{\partial y}\right) \\
& \frac{\partial \sigma_{x}}{\partial x}+\frac{\partial \tau_{x y}}{\partial y}=0 \\
& \frac{\partial \sigma_{y}}{\partial y}+\frac{\partial \tau_{x y}}{\partial x}=0 \\
& \varepsilon_{x}=\frac{1}{E}\left(\sigma_{x}-\mu \sigma_{y}\right)+\alpha \Delta T \\
& \varepsilon_{y}=\frac{1}{E}\left(\sigma_{y}-\mu \sigma_{x}\right)+\alpha \Delta T \\
& \gamma_{x y}=\frac{\tau_{x y}}{G} \\
& \varepsilon_{x}=\frac{\partial u}{\partial x} \\
& \varepsilon_{y}=\frac{\partial v}{\partial y} \\
& \gamma_{x y}=\frac{\partial v}{\partial x}+\frac{\partial u}{\partial y}
\end{aligned}
$$

Boundary conditions for thermal and mechanical equations can be obtained as,

$$
\begin{aligned}
& T=0 \text { at } x=0 \text { or } x=60 \\
& T=100 \text { at } y=0 \text { or } y=30 \\
& u=v=0 \text { at } x=0 \text { or } x=60 \\
& u=v=0 \text { at } y=0 \text { and } 6 \leq x \leq 14 \\
& u=v=0 \text { at } y=0 \text { and } 46 \leq x \leq 54
\end{aligned}
$$

$$
\begin{array}{lllll}
u=v=0 & \text { at } & y=30 & \text { and } & 6 \leq x \leq 14 \\
u=v=0 & \text { at } & y=30 & \text { and } & 46 \leq x \leq 54
\end{array}
$$

\subsection{HCAM Statement}

To approach the global optimum solution of the structure with mechanical and heat constraints, HCAM coupling CA algorithm and FE model is implemented for the topology optimization. In present study, the material relative density $x_{i}$ and the strain energy density $U_{i}$ are chosen as design variable and state variable of the optimization method, respectively. The optimization goal is minimizing the difference between average strain energy density and setting point of state variable based on the design variable. So the objective function for the HCAM topology optimization problem can be given by,

$$
\left\{\begin{array}{l}
\min \left|e_{i}\right|=\left|\bar{U}_{i}-U *\right| \\
\text { s.t. } 0<x_{\min } \leq x_{i} \leq 1, \quad i=1,2, \ldots, N
\end{array}\right.
$$

where $e_{i}$ is the difference between average strain energy density $\bar{U}_{i}$ and setting point $U^{*}$ of state variable. The subscript sequence $i$ represents the cellular number. To avoid singular matrix during the optimization process, the prescribed minimum of the material relative density should be set. Here, the prescribed minimum $x_{\min }=10^{-3} . N$ is the number of cellular number.

The average strain energy density of each cellular determined by it and its neighboring cellulars can be obtained by,

$$
\bar{U}_{i}=\frac{U_{i}+\sum_{j=1}^{\Delta N} U_{j}}{\Delta N+1}
$$

where $\Delta N$ is the number of neighboring cellulars. $U_{j}$ is the strain energy density of the neighboring cellular $\mathrm{j}$ of the $i$ cellular.

The weight approach for setting point of state variable is applied to accelerate the convergence of the topology optimization as following,

$U^{*}=\operatorname{aif} \times \frac{U_{t}}{V_{0}}$

where aif is the weight coefficient. $U_{t}, V_{0}$ are the total strain energy of $t$ iteration generarion and the initial structure volume, which can be calculated by

$$
\begin{aligned}
& U_{t}=\sum_{i=1}^{N} U_{i} v_{i} \\
& V_{0}=\sum_{i=1}^{N} v_{i}
\end{aligned}
$$

where $v_{i}$ is the volume of each cellular. Owing to the structure deformation, the strain energy density is defined as

$$
U_{i}=\frac{1}{2} \sigma^{T} \varepsilon
$$

where $\boldsymbol{\sigma}, \boldsymbol{\varepsilon}$ are the stress vector $\left[\sigma_{x}, \sigma_{y}, \tau_{x y}\right]^{T}$ and the strain vector $\left[\varepsilon_{x}, \varepsilon_{y}, \gamma_{x y}\right]^{T}$, respectively. 
During the topology optimization process, the optimization goal of state variable is obtained based on the control rules of the design variable. For the present HCAM optimization, the stress and strain results of Eqs. (1) (16) are obtained based on the relative density by the FE method, and then the relative density is renewed as CA control rules based on the strain energy density. Under preceding cycle calculations, an optimal topology structure is presented while the optimization goal is satisfied. In present study, the control rules of the design variable is expressed by reverse control strategy as

$$
x_{i}(t)=\left\{\begin{array}{lll}
1 & \text { if } \quad U_{i} \geq U^{*} \\
0.75 & \text { if } \quad U^{*}>U_{i} \geq \frac{5}{6} U^{*} \\
0.5 & \text { if } & \frac{5}{6} U^{*}>U_{i} \geq \frac{2}{3} U^{*} \\
0.25 & \text { if } & \frac{2}{3} U^{*}>U_{i} \geq \frac{1}{2} U^{*} \\
0 & \text { if } & \frac{1}{2} U^{*}>U_{i}
\end{array}\right.
$$

\subsection{Numerical Solution}

During the HCAM iteration process, the strain energy density and the material relative density are renewed alternately by FE method and CA algorithm. Considering the convergence of the calculating results and convergence efficiency, the criterion of the total mass for the topology structure is established as follow,

$$
\frac{\left|\Delta M_{n}\right|+\left|\Delta M_{n-1}\right|}{2 M_{0}} \leq 10^{-3}
$$

where $\Delta M_{n}$ is mass difference of adjacent iterations by

$$
\Delta M_{n}=M_{n}-M_{n-1}
$$

where the structure mass can be obtained by

$$
M_{n}=\sum_{i=1}^{N} x_{i} \rho v_{i}
$$

The subscript sequence $n$ represents the iteration number. So $M_{0}$ is the initial mass of the structure

For the presented HCAM, it couples the structure equation and the temperature equation for the topology optimization with mechanical and heat constraints. The solution of the temperature equation is obtained based on the material relative density. The structure equation solutes by the temperature and the material relative density are obtained. Therefore, it needs to iterate ceaselessly the structure equation and the temperature equation. It can be seen that the method is not only the coupling of structure and heat based on the thermal expansion, but also a new way of topology optimization of continuum considering the thermal effect.

\section{Results and Discussions}

To validate the developed HCAM simulation code for the structural topology optimization, test case for TOCS standard testing structure are examined using the computer method under cantilever state corresponding to Kane and Schoenauer (1996). Fig. 2 shows the physical model with fixed constraint on left vertical wall and $2000 \mathrm{~N}$ concentrated load on the right vertical wall. Table 1 lists the material properties for TOCS standard testing structure. For the validating case, both FE size and cellular size are $1 \mathrm{~mm} \times 1 \mathrm{~mm} \times 6 \mathrm{~mm}$. Higher order 8-node element is employed for the FE analysis. The von- Neumann strategy of four neighboring cellulars is implemented to renew the relative density and the weight coefficient is set as 0.48 . The initial relative density of 1.0 is applied.

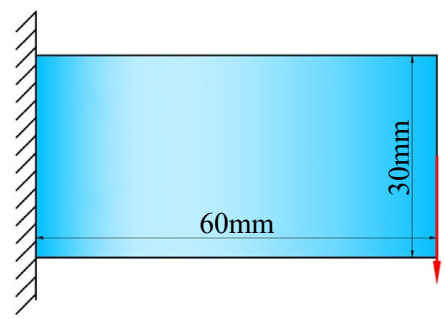

Fig. 2 Physical model for the validating case

Table 1 Material properties for TOCS standard testing structure

\begin{tabular}{ccc}
\hline Property & Unit & Value \\
\hline Density & $\mathrm{kg} / \mathrm{m}^{3}$ & 7850 \\
Elasticity modulus & $\mathrm{GPa}$ & 210 \\
Poisson ratio & --- & 0.25 \\
\hline
\end{tabular}

Fig. 3 shows the optimization results obtained by this numerical method and Kane and Schoenauer (1996). It can be seen that the results are in good agreement with each other. Further investigations present it takes 20s for the topology structure by the HCAM simulation while Kane and Schoenauer (1996) cost $24 \mathrm{hr}$ for the results. Meanwhile, there is no checkerboard phenomenon which was found in Fig. 3b for the HCAM results. Therefore, the developed HCAM code is more efficient for structural topology optimization.

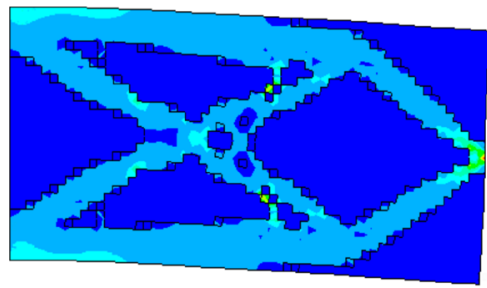

(a) Topology structure of HCAM

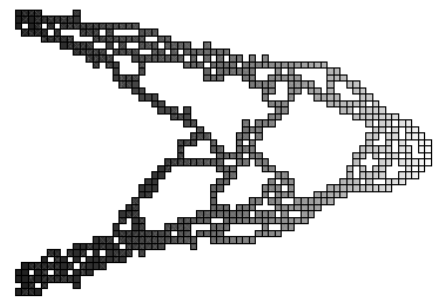

(b)Topology structure of Kane and Schoenauer (1996)

Fig. 3 Comparison of HCAM and Kane and Schoenauer (1996) for TOCS structure

Fig. 4 shows structure evolution tendency of the HCAM simulation for TOCS testing structure with mechanical and thermal constraints. Using the control rules, different material relative density of cellular unit is presented by different color in Fig. 4. The purple area represents the material relative density of 0 unit (removed area) while the light blue area is the material relative density of 1 unit (reserved area). The other colors are between the above two. According to the different colors, it can be very easy to distinguish the change trend of the material relative density after the topological optimization. For the HCAM simulation, Table 2 lists the material properties for the HCAM structure with mechanical and thermal constraints. Both FE size and cellular size are $1 \mathrm{~mm} \times 1 \mathrm{~mm} \times 6 \mathrm{~mm}$. The 4-node quadrangle element of the FE analysis is applied to structure-thermal coupling calculation. The von-Neumann 
strategy of four neighboring cellulars is used to renew the relative density. The weight coefficient and the initial relative density are given as 0.48 and 1.0, respectively. Finally, it takes 21 iterations to obtain the topology structure. It can be seen from Fig. 4 that there are two areas of lower relative density in the middle of the structure. The two areas are symmetrical about the center of the topology structure. Meanwhile, four blank areas are ultimately formed near the middle of four sides of the structure as Fig. 4d shown.

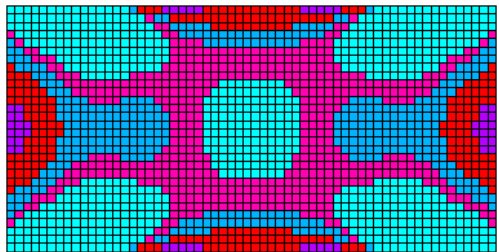

(a) $n=1$

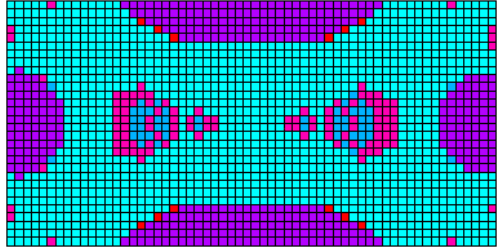

(b) $n=7$

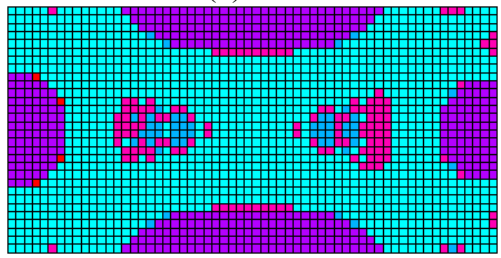

(c) $n=14$

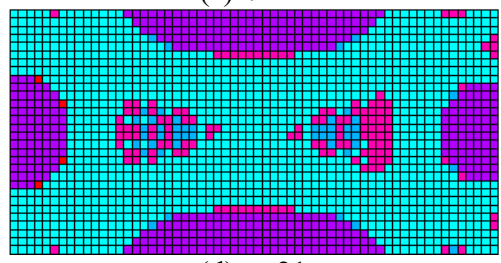

(d) $n=21$

Fig. 4 Topology structure evolution of the HCAM structure with mechanical and thermal constraints

Table 2 Material properties for the structure with mechanical and thermal constraints

\begin{tabular}{ccc}
\hline Property & Unit & Value \\
\hline Density & $\mathrm{kg} / \mathrm{m}^{3}$ & 7850 \\
Elasticity modulus & $\mathrm{GPa}$ & 210 \\
Poisson ratio & --- & 0.25 \\
Thermal expansion coefficient & --- & $1.0 \times 10^{-5}$ \\
Thermal conductivity & $\mathrm{W} /(\mathrm{m} \cdot \mathrm{K})$ & 40 \\
\hline
\end{tabular}

Fig. 5 shows stress evolution of the HCAM topology structure with mechanical and thermal constraints. The maximum stress is observed at the locations of the mechanical constraints from Fig. 5a and Fig. 5b. The minimum stress is found at four blank areas and the stress result of Fig. $5 \mathrm{~b}$ is coinciding exactly with the optimized results shown in Fig. 4d. Furthermore, the maximum stress was reduced by $19 \mathrm{Mpa}$ after the HCAM optimization. Therefore, the optimization method not only makes the structure lightweight but also decreases the structure load.

Fig. 6 shows thermal evolution of the HCAM topology structure with mechanical and thermal constraints. It can be seen that the optimized structure is mainly within a small temperature range. The temperature distribution of the optimized structure is more homogeneous than that before the optimization. Large temperature difference only presents near the vertical walls. It can eliminate most of the thermal stress in the structure. The temperature result of Fig. $6 \mathrm{~b}$ is compatible with the topology structure shown in Fig. 5b.

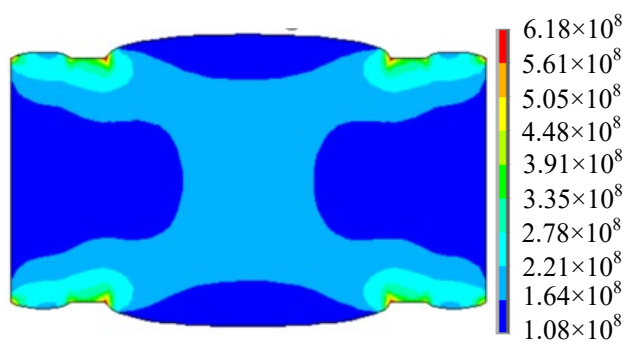

(a) $n=0$

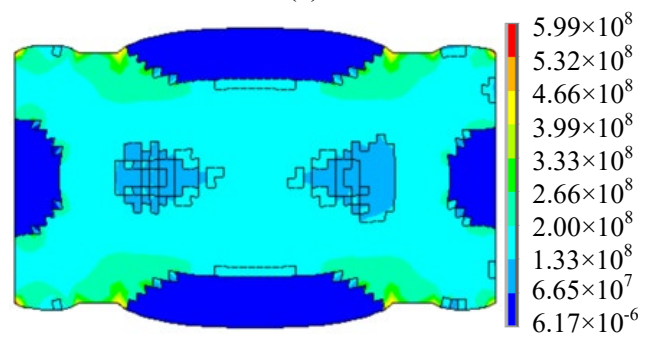

(b) $n=21$

Fig. 5 Stress evolution of topology structure with mechanical and thermal constraints

Fig. 7 shows structure performance evolution of the HCAM topology structure with mechanical and thermal constraints during the iteration process. The performance index PI represents the product of initial cellular maximum strain energy density of and cellular mass relative to the product of the current iteration. It can be seen from Fig. 7 that the $P I$ index increases gradually after a sharp change and finally level off until the convergence. It means that the product of cellular maximum strain energy density of and cellular mass decreases during the optimization process. The optimization measure gradually realize its maximum potential at the convergence iteration. Meanwhile, the cellular mass ratio $F$ decreases to the minimum as the iteration process. It reveals the topology structure is obtained as the optimization.

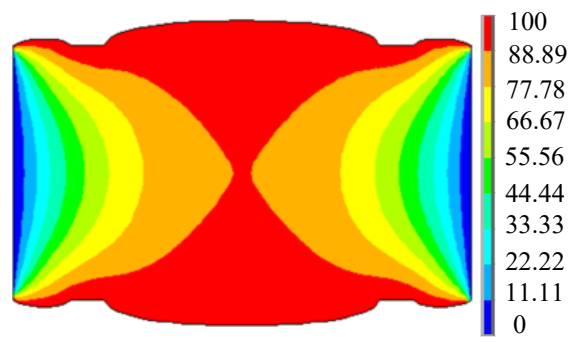

(a) $n=0$

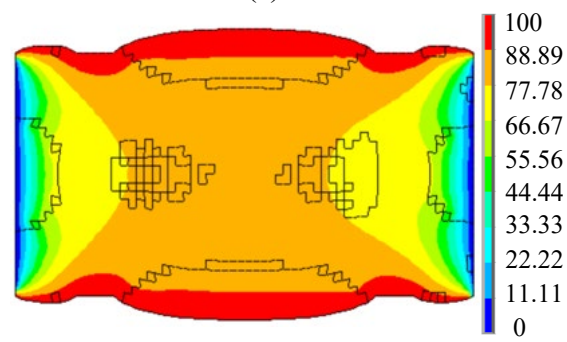

(b) $n=21$

Fig. 6 Thermal evolution of topology structure with mechanical and thermal constraints 


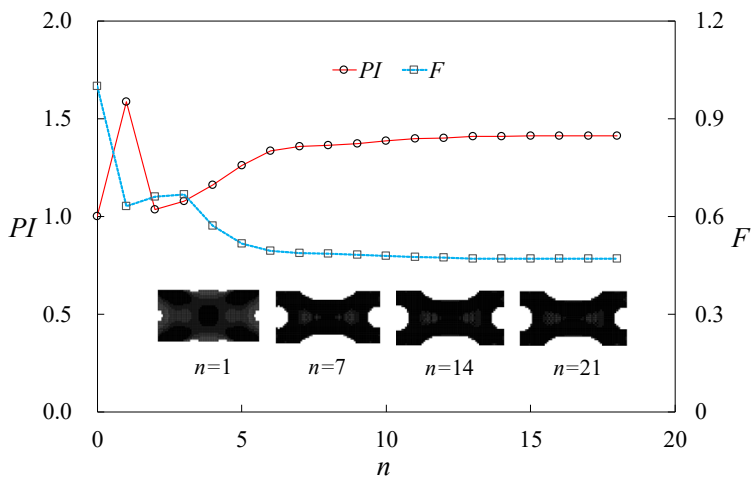

Fig. 7 structure performance evolution of topology structure with mechanical and thermal constraints

\section{CONCLUSIONS}

A hybrid cellular automaton model combined with FE method for structural topology optimization with mechanical and heat constraints as well as thermal stress into account has been developed. The model has been validated by comparing topology structure with the reference results. The structure evolution, stress evolution and thermal evolution of topology optimization with mechanical and heat constraints have been presented. The numerical results show the optimization method not only makes the structure lightweight but also decreases the structure load. Furthermore, the temperature distribution of the optimized structure is more homogeneous and most of the thermal stress in the structure is eliminated.

\section{ACKNOWLEDGEMENTS}

This work is supported by Chinese National Natural Science Foundation Grant 51605253, Zhejiang Postdoctoral Research Project zj20180077, Zhejiang Basic Public Welfare Research Project LGG18E050014 and Scientific Research Starting Project of SWPU 2018QHZ031.

\section{NOMENCLATURE}

$\begin{array}{ll}c_{p} & \text { specific heat }\left(\mathrm{kJ} \cdot \mathrm{kg}^{-1} \cdot \mathrm{K}^{-1}\right) \\ E & \text { modulus of elasticity }(\mathrm{Pa}) \\ G & \text { modulus of shear }(\mathrm{Pa}) \\ t & \text { tim }(\mathrm{s}) \\ T & \text { temperature }(\mathrm{K}) \\ u & \text { displacement in } \mathrm{x} \text { direction }(\mathrm{m}) \\ v & \text { displacement in y direction }(\mathrm{m}) \\ x & x \text { direction } \\ y & y \text { direction } \\ \text { Greek Symbols } & \\ \sigma_{x} & \text { normal stress }(\mathrm{Pa}) \\ \sigma_{y} & \text { normal stress }(\mathrm{Pa}) \\ \tau_{x y} & \text { shear stress }(\mathrm{Pa}) \\ \varepsilon_{x} & \text { normal strain } \\ \varepsilon_{y} & \text { normal strai } \\ \gamma_{x} & \text { shear strain } \\ \gamma_{y} & \text { shear strain } \\ \mu & \text { poisson ratio } \\ \alpha & \text { coefficient of linear expansion }\left(\mathrm{K}^{-1}\right)\end{array}$

\section{REFERENCES}

Bendsoe, M. P., Diaz, A., Lipton, R., and Taylor, J. E., 2010, “Optimal Design of Material Properties and Material Distribution for Multiple Loading Conditions", International Journal for Numerical Methods in Engineering, 38(7), 1149-1170. http://dx.doi.org/10.1002/nme.1620380705.

Bandi, P., Schmiedeler, J., and Tovar, A., 2013, "Design of Crashworthy Structures with Controlled Energy Absorption in the Hybrid Cellular Automaton Framework", Journal of Mechanical Design, 135(9), 12811293. http://dx.doi.org/10.1115/1.4024722.

Czubacki, R., and Lewiński, T., 2015, “Topology Optimization of Spatial Continuum Structures: Made of Nonhomogeneous Material of Cubic Symmetry", Journal of Mechanics of Materials \& Structures, 10(4), 519535.

http://dx.doi.org/10.2140/jomms.2015.10.519.

Du, Y., Chen, D., Xiang, X.B., Tian, T. Q., and Zhang, Y., 2013, "Topological Design of Structures Using a Cellular Automata Method", Cmes Computer Modeling in Engineering \& Ences, 94(1), 53-75.

Eschenauer, H. A., and Olhoff, N., 2001, "Topology Optimization of Continuum Structures: A Review”, Applied Mechanics Reviews, 54(4), 1453-1457.

http://dx.doi.org/10.1115/1.1388075.

Kane, C., and Schoenauer, M., 1996, "Topological Optimum Design Using Genetic Algorithms", Proceedings of Control and Cybernetics, 25(5), 1-15.

Kushnir, R., 2009, "Thermal Stresses-advanced Theory and Applications ", Journal of Thermal Stresses, 158(1), 341-369. http://dx.doi.org/10.1007/978-1-4020-9247-3.

Lee, S., Tovar, A., 2013, "Topology Optimization of Piezoelectric Energy Harvesting Skin Using Hybrid Cellular Automata", Journal of Mechanical Design Transactions of the ASME, 135(3), 1-11. http://dx.doi.org/ 10.1115/1.4023322.

Min, S. K., Sang, R. K., and Han, S. Y., 2007, “Topology Optimization of a PCB Substrate Considering Mechanical Constraints and Heat Conductivity", Journal of Mechanical Science \& Technology, 21(12), 2041-2047.

http://dx.doi.org/10.1007/bf03177462.

Pettermann, H. E., Reiter, T. J., and Rammerstorfer, F. G., 1997, "Computational Simulation of Internal Bone Remodeling", Archives of Computational Methods in Engineering, 4(4), 295-323. http://dx.doi.org/ 10.1007/BF02737117.

Sigmund, O., 2009, "Manufacturing Tolerant Topology Optimization", Acta Mechanica Sinica, 25(2), 227-239.

http://dx.doi.org/10.1007/s10409-009-0240-z.

Sanaei, E., and Babaei, M., 2011, "Cellular Automata in Topology Optimization of Continuum Structures", International Journal of Engineering Science \& Technology, 3(4), 27-41. http://dx.doi.org/10.4314/ijest.v3i4.68538.

Sanaei, E., and Babaei, M., 2012, “Topology Optimization of Structures Using Cellular Automata with Constant Strain Triangles", International Journal of Civil Engineering, 10(3), 179-188.

Salonitis, K., 2017, “A Hybrid Cllular Automata-finite Element Model for the Simulation of the Grind-hardening Process", International Journal of Advanced Manufacturing Technology, 1, 1-7. http://dx.doi.org/10.1007/s00170-017-0829-5. 
Tovar, A., Patel, N., Kaushik, A. K., 2004, "Hybrid Cellular Automata: A Biologically-inspired Structural Optimization Technique", Proceedings of 10th AIAA/ISSMO Multidisciplinary Analysis and Optimization Conference, New York, NYC.

http://dx.doi.org/10.2514/6.2004-4558.

Tovar, A., Quevedo, W. I., Patel, N. M., and John, E. R., 2005, "Hybrid Cellular Automata with Local Control Rules: A New Approach to Topology Optimization Inspired by Bone Functional Adaptation", Proceedings of 6th World Congresses of Structural and Multidisciplinary Optimization, Rio de Janeiro, Brazil.

Tovar, A., Patel, N. M., Niebur, G. L., Mihir S., and John E. R., 2006, "Topology Optimization Using a Hybrid Cellular Automaton Method with Local Control Rules", Journal of Mechanical Design, 128, 12051216.

http://dx.doi.org/10.1115/1.2336251.
Tovar, A., Patel, N. M., Kaushik, A. K., and John, E. R., 2007, "Optimality Conditions of the Hybrid Cellular Automata for Structural Optimization", AIAA Journal, 45(3), 673-683. http://dx.doi.org/10.2514/1.20184.

Takezawa, A., Yoon, G. H., and Jeong, S. H., 2014, "Structural Topology Optimization with Strength and Heat Conduction Constraints", Computer Methods in Applied Mechanics \& Engineering, 276(8), 341361.

http://dx.doi.org/10.1016/j.cma.2014.04.003.

Zhu, Z., and Liu, C., 2000, "Micromachining Process Simulation Using a Continuous Cellular Automata Method", Journal of Microelectromechanical Systems, 9(2), 252-261.

http://dx.doi.org/10.1109/84.846706. 